\title{
Dolor abdomino-pélvico en ginecología
}

\section{Abdominal-pelvic pain in gynaecology}

\author{
R. Ezcurra, N. Lamberto, V. Peñas
}

\section{RESUMEN}

El dolor abdomino-pélvico es una entidad compleja, a veces de difícil diagnóstico, que requiere un análisis profundo para determinar sus causas y el tratamiento más adecuado. Implica a diversas vísceras por lo que frecuentemente el abordaje debe ser multidisciplinar y en ocasiones exige una actuación rápida ya que está en juego la vida de la paciente

Se han expuesto en este trabajo sólo las causas de origen ginecológico pues entendemos que el resto de patologías corresponde explicarlas a otras especialidades.

Se expone un cuadro de diagnóstico diferencial entre las causas más frecuentes de dolor pélvico de origen ginecológico.

Palabras clave. Dolor pélvico. Torsión de ovario. Embarazo ectópico. Enfermedad pélvica inflamatoria. Endometriosis.

\begin{abstract}
Abdominal-pelvic pain is a complex entity, at times difficult to diagnose, which requires a deep analysis to determine its causes and the most suitable treatment. It involves different viscera which is why its management must frequently be multidisciplinary and on occasion it requires rapid action as the patient's life is at stake.

This article sets out only the causes with a gynaecological origin since, in our understanding, it corresponds to other specialities to explain the rest of the pathologies.

A diagnostic table is set that differentiates amongst the most frequent causes of pelvic pain with a gynaecological origin.
\end{abstract}

Key Words. Pelvic pain. Ovarian torsion. Ectopic pregnancy. Pelvic inflammatory disease. Endometriosis.
Servicio de Obstetricia y Ginecología. Hospital Virgen del Camino. Pamplona.
Correspondencia:

Ricardo Ezcurra Irure

Avda. Sadar, 42

31192 Mutilva Baja (Navarra)

Tfno. 647559863

E-mail: rezcurri@cfnavarra.es 


\section{INTRODUCCIÓN}

El dolor abdominopélvico agudo es una de las causas más frecuentes de consulta ginecológica y la causa más frecuente de hospitalización.

La valoración inicial más importante que se debe realizar es determinar si se trata de un abdomen agudo quirúrgico y si precisa hospitalización inmediata. Es importante descartar la gestación al comenzar la evaluación de la paciente (considerar posibilidad de gestación ectópica rota, que puede convertirse en una situación de riesgo vital).

Dentro de los posibles diagnósticos se presentan: gestación ectópica, torsión de quiste de ovario (la importancia de su diagnóstico precoz radica en una rápida intervención para preservar el ovario y la trompa), dismenorrea, endometriosis y los miomas.

El diagnóstico oportuno del dolor pélvico agudo es de importancia crítica porque el retraso podría aumentar la morbimortalidad. La historia precisa es clave para establecer el diagnóstico correcto. Es necesario verificar la fecha y el carácter de los dos últimos periodos menstruales y la presencia de hemorragia o secreción anormal.

\section{DOLOR PÉLVICO AGUDO}

Se debe tener en cuenta el comienzo, carácter, localización y patrón de irradiación del dolor y correlacionarlos con los cambios (micción, defecación, relaciones sexuales, actividad física...), la regularidad de los periodos menstruales, la posibilidad de embarazo, la presencia de sangrado vaginal o flujo, antecedentes medicoquirúrgicos. Una historia reciente de dispareunia o dismenorrea es sugestiva de patología pélvica. Las causas más frecuentes de dolor pélvico agudo en las mujeres incluye: enfermedad inflamatoria pélvica (EIP), masas o quistes anexiales con torsión, rotura o sangrado, gestación ectópica, endometritis o degeneración, infarto o torsión del mioma.

Cuando se presenta una mujer con dolor abdominopélvico, la valoración inicial más importante es determinar si se trata de un abdomen agudo quirúrgico. Las dos siguientes consideraciones son averiguar si se encuentra embarazada, (considerar la posibilidad de embarazo ectópico) y si precisa hospitalización inmediata. Hay que hacer una valoración rápida para identificar pacientes que requieren intervención quirúrgica urgente, incluyendo anamnesis, si es posible, y exploración física, incluyendo examen pélvico. Pueden presentarse signos de gravedad que indican necesidad de cirugía urgente (inestabilidad hemodinámica: hipotensión, confusión, diaforesis, obnubilación...).

Es importante determinar los antecedentes del dolor: cómo y en qué momento se inició éste: la presencia de síntomas gastrointestinales (ej: anorexia, náuseas, vómitos, estreñimiento relativo o pertinaz, flatulencia); de síntomas urinarios (ej: sensación urgente de orinar, micción frecuente, hematuria y disuria y signos de infección (fiebre, escalofrío).

\section{CAUSAS DE DOLOR PÉLVICO} Dolor agudo: enfermedad o disfunción
ginecológica

- Complicación del embarazo

- Embarazo ectópico (EE) roto

- Amenaza de aborto (AA) o aborto incompleto

- Degeneración de leiomioma

- Infecciones agudas

- Endometritis

- Enfermedad inflamatoria pélvica

- Absceso tuboovárico

- Trastornos de los anexos

- Quiste ovárico funcional hemorrágico

- Torsión de anexo

- Quiste paraovario torsión

- Rotura quiste ovárico (funcional o neoplásico: dermoide/endometrioma...)

\section{Dolor pélvico recurrente}

- Dolor periovulatorio (Mittelschmerz)

- Dismenorrea primaria/secundaria

\section{Causas gastrointestinales}

- Gastroenteritis

- Apendicitis

- Obstrucción intestinal

- Diverticulitis

- Enfermedad inflamatoria intestinal

- Síndrome colon irritable 


\section{Causas geritourinarias}

- Cistitis

- Pielonefritis

- Litiasis ureteral

\section{Causas musculoesqueléticas}

- Hematoma pared abdominal

- Hernia inguinal

\section{CAUSAS GINECOLÓGICAS (Tabla 1)}

Tabla 1. Cuadro de diagnóstico diferencial abdomen agudo ginecológico.

\begin{tabular}{llll}
\hline \multicolumn{1}{c}{ Sospecha diagnóstica } & \multicolumn{1}{c}{ Clínica } & \multicolumn{1}{c}{ Confirmación } & \multicolumn{1}{c}{ Tratamiento } \\
\hline EIP & $\begin{array}{l}\text { Fiebre, dolor, flujo vaginal } \\
\text { anómalo }\end{array}$ & $\begin{array}{l}\text { Bacteriológico } \\
\text { Laparoscopia }\end{array}$ & $\begin{array}{l}\text { Antibioterapia } \\
\text { Cirugía }\end{array}$ \\
\hline Ectópico & $\begin{array}{l}\text { Amenorrea, dolor, } \\
\text { sangrado vaginal, } \\
\text { hemoperitoneo }\end{array}$ & $\begin{array}{l}\text { Hemograma } \\
\text { Prueba de embarazo } \\
\text { Laparoscopia }\end{array}$ & $\begin{array}{l}\text { Laparoscopia vs } \\
\text { Laparotomía }\end{array}$ \\
\hline Quiste anexial & Masa anexial & Ecografía & \\
& Tacto doloroso & & Laparoscopia vs \\
& Menometrorragia & Ecografía & Expectante vs \\
Mioma & Útero irregular & & Laparotomía \\
\hline Infección orina & Fiebre, dolor lumbar/ & Urocultivo & Antibioterapia \\
& suprapúbica & & \\
\hline
\end{tabular}

\section{Ováricas}

- Quiste ovárico complicado (hemorragia, torsión, rotura)

- Rotura folicular

\section{Tubáricas}

- Embarazo ectópico

- Torsión anexial

$$
\text { - EIP }
$$

\section{Uterinas}

- EE intersticial o en cuerno

- Mioma complicado

- Adenomiosis

- Aborto en curso

\section{Otras causas}

- Porfiria aguda

- Tromboflebitis pélvica

- Aneurisma

- Angina abdominal

\section{DIAGNÓSTICO DEL DOLOR PÉLVICO AGUDO}

Para un correcto diagnóstico es importante la historia clínica, tipo de dolor, da- tos clínicos, analíticos y pruebas de diagnóstico completas. Se debe determinar su relación con la menstruación y ovulación o coito.

- Historia y examen físico

- Hemograma y coagulación

- Orina, sedimento

- Prueba de embarazo (orina/suero)

- Cultivo cervical (Gonococo/Chlamydia)

- Ecografía pélvica

- Si prueba de embarazo +: descartar EE

- Tumoración pélvica de diagnóstico incierto

- Radiografía abdominal del tubo digestivo si predominan síntomas digestivos

- TAC: valorar tumoración retroperitoneal, o abscesos del tubo digestivo

- Laparoscopia diagnóstica:

- Abdomen agudo de causa no filiada

- Aclarar la naturaleza de una tumoración dudosa de un anejo 
- Delimitar si un embarazo es intra o extrauterino

- Mejorar la precisión del diagnóstico si se sospecha salpingooforitis

- Laparotomía (laparoscopia relativamente contraindicado)

- Peritonitis

- Íleo grave

- Obstrucción intestinal

\section{EMBARAZO ECTÓPICO}

Embarazo ectópico es una de las urgencias ginecológicos más frecuentes, constituyendo la primera causa de muerte materna por shock hemorrágico.

La incidencia es del 1\%, en aumento (EIP, dispositivo intrauterino -DIU-, tratamiento de reproducción asistida (TRA), cirugía conservadora, mayor edad...). Gestación heterotópica (1/30.000).

\section{Manifestaciones clínicas}

Aparecen habitualmente con 6 ó 7 semanas de amenorrea, aunque puede ocurrir más tarde. Puede presentarse desde asintomático hasta shock hipovolémico y muerte.

Los síntomas clásicos son (EE íntegro o si rotura) ${ }^{1,2}$ :

- Dolor abdominal: es el síntoma mas importante, en el $99 \%$ casos

- Amenorrea en el 75\%

- Sangrado vaginal (escasa cantidad, intermitente, oscuro) en el $56 \%$

Un EE debe ser sospechado en cualquier mujer en edad reproductiva con estos síntomas, especialmente con factores de riesgo, para poder tratar de forma menos agresiva (aproximadamente la mitad pasan desapercibidos en la primera visita).

Factores de riesgo ${ }^{3}$ :

- Alto riesgo

- EE previo

- Cirugía tubárica previa (aumenta riesgo un 5-10\%)

- Ligadura de trompas (x 20 veces )

- Patología tubárica/endometriosis.

- Dietilestilbestrol intraútero

- DIU (más frecuente en "liberador de hormonas")
- Riesgo moderado

- Infertilidad. Fecundación in vitro (FIV): (incidencia 1-3\%) ${ }^{4}$

- Cervicitis previa

- Historia de EIP (Chlamydia, riesgo $\mathrm{x} 6)^{5}$

- Múltiples parejas sexuales

- Tabaco

- Bajo riesgo

- Cirugía abdominopélvica previa (adherencias)

- Comienzo precoz de relaciones sexuales

Es importante realizar un test de embarazo en mujeres con dolor abdominal o sangrado vaginal para enfocar la evaluación posterior.

Amenorrea más dolor abdominal con presencia o ausencia de sangrado vaginal son síntomas de complicación del embarazo precoz (amenaza de aborto, rotura o torsión de cuerpo lúteo, degeneración de leiomioma uterino, EE.).

\section{TEST DIAGNÓSTICOS RECOMENDADOS}

\section{Ecografía transvaginal}

El test Eco transvaginal es el más útil para determinar la localización del saco. Posee alta sensibilidad y especificidad. Si hay rotura aparece líquido libre en abdomen.

Detecta la presencia o ausencia de un saco gestacional dentro o fuera del útero y así establece el diagnóstico. Una masa anexial compleja + test de embarazo positivo y útero vacío es altamente sugestiva de un EE y es la alteración ecográfica más común (E: 99,9\%, VPP 96,7\%, VPN 99,4\%).

\section{BhCG}

La combinación del Eco transvaginal y la gonadotropina coriónica ßh permite el diagnóstico definitivo en casi todos casos, de forma muy precoz, permitiendo tratamientos menos invasivos que la escisión quirúrgica ${ }^{7}$.

Puede ser detectada en suero y orina 8 semanas tras aparición de pico LH. Aumenta más despacio en la mayoría, pero no 
siempre (EE y gestaciones no viables ${ }^{8}$ o a veces similar).

La medida de BhCG cada 72 horas en vez de cada 48 horas es más practica ${ }^{9}$. Un aumento normal de BhCG se debe evaluar con ecografía (si BhCG mayor de 1.500) pudiendo diagnosticarse intrauterino o ectópico. Si la ßhCG no se dobla tras 72 horas, es posible afirmar que se trata de una gestación intrauterina normoevolutiva (anembrionado, aborto tubárico, aborto, EE resuelto espontáneamente). Un descenso de BhCG es más consistente con una gestación fallida; el descenso es más lento con un EE, medida semanal hasta BhCG (-).

A partir de 1.500 (2.000 UI/l) de ßhCG se debe poder visualizar el saco con eco ${ }^{10}$ (la ecografía no es sensible para determinar la localización cuando BhCG es menor). La ausencia de saco intrauterino con este nivel de BhCG sugiere EE o no viable o puede representar una gestación múltiple por lo que se repite ecografía y $\mathrm{BhCG}$ en 48 horas.

Un EE puede ser diagnosticado si la concentración de ßhCG está aumentada o "plateau" y no se visualiza por ecografía.

Otros test diagnósticos son: nivel de progesterona en suero, eco-Doppler, laparoscopia, RMN, culdocentesis, pero no añaden información clínica adicional útil.

El tratamiento se puede orientar desde un punto de vista médico o quirúrgico. Si se sospecha rotura de trompa o existe inestabilidad hemodinámica, el tratamiento de elección es laparoscopia con salpinguectomía. Si no existe rotura de trompa se puede considerar un tratamiento más conservador con salpingostomía (apertura de trompa) y extracción del saco; posteriormente se deben realizar controles de ßhCG has su negativización. Pero si la paciente esta sintomática, la ßhCG es menor de 5.000 UI y la trompa menor de $3 \mathrm{~cm}$ se puede intentar tratamiento médico con 50 mg de metotrexate en dosis única i.m. y posterior control de BhCG hasta su negativización.

Aunque el factor más importante para el pronóstico de fertilidad futura es el diagnóstico precoz, es importante conocer el estado de la trompa contralateral y el antecedente de la cirugía tubárica.

\section{QUISTE OVÁRICO ROTO}

Esta situación es frecuente en edad reproductiva; más frecuente en el anejo derecho. Puede ser asintomático o asociado con el inicio repentino de dolor abdominal unilateral, y que comienza durante una actividad física o relación coital. Puede estar acompañado de un ligero sangrado vaginal. Según la naturaleza del quiste, puede permanecer asintomático (seroso o mucinoso) o produce dolor intenso (dermoide: peritonitis química).

Lo más importante es excluir el EE roto (hemoperitoneo) por la necesidad de cirugía urgente.

Hay que efectuar prueba de embarazo, hemograma, grupo y Rh, análisis de orina; ecografía: (sensibilidad: $85-100 \%)^{11}$; una masa anexial con líquido en pelvis sugiere rotura, pero no es diagnóstico. Evitar culdocentesis, paracentesis y marcadores tumorales.

Si la rotura no es complicada hacer manejo ambulatorio y analgesia oral a demanda. El líquido se reabsorbe en 24 horas y los síntomas mejoran en unos días (posible cirugía posterior si quiste persistente, o crece, o sospecha de malignidad).

Si se complica con hemoperitoneo es preciso hospitalización con reemplazo de fluido, constantes vitales, hematocrito seriado y repetir ecografía. Una vez que cesa, tarda varias semanas en reabsorberse. Si la paciente está inestable se valorará laparoscopia (laparotomía).

Si existe rotura de quiste dermoide (poco frecuente) se producirá peritonitis química, que desarrolla formación de adherencias y dolor pélvico crónico.

\section{TORSIÓN DE ANEJOS}

Es la quinta emergencia ${ }^{12}$ ginecológica en frecuencia $(2,7 \%)$ tras EE, rotura de cuerpo lúteo, EIP y apendicitis. Representa un $15 \%$ de las masas anexiales intervenidas. Afecta a mujeres de todas edades, (80\% en edad reproductiva). El riesgo aumenta en embarazadas y con TRA (8\%) en ovarios con síndrome de hiperestimulación ovárica. Sin embargo, su diagnóstico puede ser un reto, debido a síntomas poco específicos. Un diagnóstico oportuno es impor- 
tante para preservar la función del ovario y prevenir secuelas (necrosis, hemorragia, infarto, peritonitis...). Una torsión tubárica aislada es muy infrecuente, generalmente ocurre con patología tubárica o infección. El aumento brusco de presión abdominal facilita la torsión.

La etiología más frecuente son los quistes de ovario ${ }^{13}$. El $90 \%$ son benignos. A mayor tamaño del quiste, es más probable la torsión.

La clínica más común es un inicio brusco de dolor abdominal bajo, a menudo asociado con crisis de náuseas o vómitos. La fiebre como marcador de necrosis causa leucocitosis (un cuadro de dolor crónico con crisis de dolor severo puede asociarse a una torsión intermitente) $)^{14}$. El diagnóstico definitivo se realiza en la cirugía (sólo un $38 \%$ de casos con diagnóstico correcto previo).

Mediante ecografía, el hallazgo más común es un agrandamiento heterogéneo de un ovario, sin embargo un ovario normal no lo descarta. La utilidad del Doppler es controvertida $^{15}$, hallando disminución $\mathrm{o}$ ausencia de flujo venoso. La hemorragia aumenta la sospecha de torsión.

Este cuadro clínico debe considerarse ante una mujer con dolor abdominal y masa anexial, descartando EE, rotura de quiste hemorrágico, EIP, leiomioma,...

Ante la sospecha, es oportuna una evaluación en quirófano, y el manejo posterior dependerá de los hallazgos y la edad de la paciente. Se ha demostrado la seguridad y la eficacia de la conservación del ovario, cuando es posible ${ }^{16}$. La mayoría recupera su forma y función previa, incluso si parecen isquémicos tras la destorsión por la laparoscopia ${ }^{17}$.

\section{ENFERMEDAD INFLAMATORIA PÉLVICA}

Es una infección aguda del tracto genital superior (útero-trompas-ovarios). A menudo se acompaña de afectación de los órganos pélvicos vecinos (endometritis, salpingitis, ooforitis, peritonitis, perihepatitis y absceso tuboovárico); el endometrio y el ovario son menos susceptibles a la infección que la trompa, pero pueden ser el foco de infección.
Es una infección adquirida sexualmente en la mayoría de casos y menos frecuentemente es causado por procedimientos médicos, embarazo y otros procesos abdominales primarios. Representa un espectro dentro de la infección. No hay un único diagnóstico "gold-estándar" y el valor del diagnostico clínico tiene la mayor importancia.

\section{Síntomas}

El dolor abdominal inferior es el síntoma principal de presentación, aunque la característica del dolor puede ser bastante sutil. El comienzo reciente del dolor que empeora durante el coito puede ser el único síntoma y el inicio durante o justo después de la menstruación es particularmente sugestivo ${ }^{18}$. El dolor abdominal es normalmente bilateral y raramente de más de 2 semanas de duración.

En un tercio de los casos se añade sangrado uterino anormal ${ }^{19}$. Aparecen flujo vaginal, uretritis y fiebre que pueden estar asociados, pero no son ni sensibles ni específicos para el diagnostico. La EIP es menos probable si existen síntomas referentes al tracto urinario o digestivo.

Son factores de riesgo para enfermedades de transmisión sexual: edad inferior a 25 años, precocidad sexual, no métodos de barreras, nuevas o múltiples o parejas sexuales sintomáticas, anticonceptivos orales y ectopia cervical.

Hay que evaluar a la paciente y los factores de riesgo que aumentan la probabilidad de EIP como son: episodios previos de EIP, relaciones sexuales durante la regla, ducha vaginal, vaginosis bacteriana y DIU.

En el examen físico sólo la mitad tienen fiebre y dolor difuso abdominal. Flujo purulento endocervical y/o dolor a la movilización cervical y dolor anexial en la exploración bimanual es altamente sugestiva de EIP. Útero y anejos: punto de máximo dolor. La lateralización del dolor anexial es poco común en la EIP; la existencia de una masa anexial palpable puede representar absceso tuboovárico y otros procesos.

\section{Consideraciones del diagnóstico}

La EIP representa un espectro de enfermedad clínica desde endometritis a sepsis 
intraabdominal. A pesar de que la laparoscopia tiene sustancial valor en la confirmación del diagnóstico de EIP, no es suficientemente sensible para ser considerada una "gold-estándar" en el diagnóstico.

\section{Criterios diagnósticos}

El grado de sospecha debe ser alto especialmente en mujeres adolescentes aunque nieguen relaciones sexuales. Un mínimo de criterios para el tratamiento empírico ha sido recomendado por la CDC para reducir la posibilidad de perder o retrasar el diagnóstico ${ }^{20}$.

Se aconseja tratamiento empírico en mujeres con dolor abdominal que tienen al menos uno de los siguientes criterios:

- Movilización cervical dolorosa o dolor uterino/anexial

- Temperatura mayor de $38,3^{\circ} \mathrm{C}$

- Leucocitosis con desviación izqda

- Flujo anormal mucopurulento cervical o vaginal

- Leucocitos en frotis vaginal

- Aumento de la velocidad de sedimentación globular

- Aumento de la proteína $C$ reactiva

\section{Test diagnósticos}

Incluye analítica completa buscando signos de inflamación, frotis de las secreciones vaginal o cervical, cultivos y estudios de imagen.

Siempre hay que comenzar con una prueba de embarazo para descartar EE. El hemograma es poco útil ya que sólo la mitad tienen leucocitosis ${ }^{21}$.

El examen al microscopio del flujo puede ofrecer información útil, si el Gram es + para diplococos la probabilidad de EIP es muy alta; si es negativo es poco útil. Test chlamydia y gonococo, sedimento de orina, PCR. La ecografía resulta un test que ofrece un diagnóstico definitivo de EIP según la CDC.

\section{Recomendación}

Se debe tener un umbral bajo para el diagnóstico de EIP, y las mujeres jóvenes sexualmente activas con la combinación de dolor abdominal bajo, dolor a la movilización cervical o anexial deben recibir tratamiento empírico. La especificidad de estos criterios puede aumentar por la presencia de fiebre, flujo vaginal/cervical anormal, aumento de VSG y/o PCR y la demostración de infección por gonococo/chlamydia.

Incluso mujeres con hallazgos mínimos deben ser tratadas. El diagnóstico diferencial es extenso, sin embargo, el tratamiento antibiótico no debe ser retrasado, cuando la sospecha es alta.

\section{ABSCESO TUBO-OVÁRICO}

Es una secuela de la salpingitis aguda y suelen ser bilaterales. Signos y síntomas similares a salpingitis aguda, pero frecuentemente dolor y fiebre mayor a 1 semana. Absceso roto: urgencia quirúrgica por posible shock tóxico por gram negativo

Al hacer un examen bimanual se aprecian tumoraciones bilaterales, fijas muy firmes y de gran sensibilidad.

Es preciso confirmar el diagnóstico mediante ecografía. Hacer diagnóstico diferencial de una tumoración unilateral: torsión de anejo, endometrioma, quiste de ovario, absceso periapendicular. Si el examen y la ecografía no dan un resultado definitivo: laparoscopia / laparotomía.

En el tratamiento hay que diferenciar entre absceso no roto aplicando posible tratamiento con antibióticos intravenosos, o roto con peritonitis difusa: taquicardia, hipersensibilidad de rebote. Laparoscopia/ Laparotomía exploradora: resección del tejido infectado.

\section{ENDOMETRITIS}

\section{Endometritis aguda}

Es un componente común de EIP, dolor pélvico y sangrado vaginal anormal, frecuentemente con fiebre. En población no gestante normalmente va precedido de EIP tras ETS o de proceso ginecológico invasivo. Procedimientos ginecológicos transcervicales son una causa poco frecuente por lo que no se recomienda realizar test para Chlamydia, y gonorrea previos. Tras interrupción de embarazo, se recomienda pro- 
filaxis antibiótica. Se sugiere su uso previo a histerosalpingografía o histerosonografía en mujeres con historia de EIP y después de proceso en que se halle hidrosalpinx.

En el $70-90 \%$ de las laparoscopias por salpingitis existe endometritis. La endometritis puede estár fuertemente asociada a Chlamydia, y más débilmente también existe asociación con gonococo y vaginosis bacteriana

\section{Leiomioma uterino (tumor del músculo liso uterino)}

Es un tumor del músculo liso del útero que puede causar molestias cuando comprime vejiga, recto adyacente o ligamentos de sostén de útero. Es raro el dolor pélvico atribuible al mioma, pero es posible si degenera o se torsiona.

La degeneración de los miomas es secundaria a la pérdida de riego sanguíneo, causado por crecimiento rápido relacionado con el embarazo. En una mujer no embarazada se confunde con salpingooforitis subaguda.

Existe el leiomioma subseroso pediculado que produce torsión y necrosis isquémica y el leiomioma submucoso pediculado que se manifiesta con dolor tipo cólico "trabajo de parto" y hemorragia.

Como signos aparecen: hipersensibilidad abdominal a la palpación e hipersensibilidad de rebote leve localizada, aumento de temperatura y leucocitosis.

Para diagnóstico y tratamiento es preciso realizar:

- Ecografía

- Degeneración: analgésicos y observación

- Torsión subseroso pediculado: laparoscopia, no indispensable intervención

- Torsión submucoso con dolor y hemorragia: histeroscopia

\section{ENDOMETRIOSIS}

Se define así a la presencia de glándulas endometriales y estroma fuera de la cavidad endometrial y del músculo uterino.

El dolor es el síntoma mas común asociado a la endometriosis; aproximadamen- te $3 / 4$ partes de los pacientes sintomáticas experimentan dolor pélvico y/o dismenorrea $^{22}$, el cual puede ser crónico, pero a menudo es más severo durante la regla o durante la ovulación. Se puede presentar con subfertilidad, dispareunia profunda, síntomas intestinales o urinarios cíclicos, sangrado uterino anormal y fatiga crónica.

El dolor pélvico ha sido atribuido al sangrado activo, producción de sustancias e irritación de nervios pélvicos. El grado de endometriosis no se correlaciona con la presencia de síntomas severos (los síntomas pueden estar más relacionados con la reacción inflamatoria peritoneal local que con el volumen de implantes). El lugar y profundidad de la implantación se correlaciona con el tipo y la severidad de los síntomas $^{23-25}$. El dolor pélvico es más común en mujeres con implantes infiltrantes profundos ${ }^{26}$.

El diagnóstico de endometriosis debe hacerse por visualización directa de los implantes.

Puede probarse la supresión hormonal ovárica ("pseudomenopausia") como tratamiento y como medio para confirmar la correlación entre el dolor actual y el diagnóstico subyacente.

No existe tratamiento que cure la enfermedad sólo se puede realizar tratamientos sintomáticos y paliativos: laparoscopia y laparotomía con cistectomía versus ovariectomía. Aplicar tratamiento expectante si es pequeño $(<3 \mathrm{~cm})$ y no está roto.

\section{DOLOR PÉLVICO PERIOVULATORIO (Mittelschmerz)}

El dolor pélvico periovulatorio también denominado Mittelschmerz es un dolor unilateral menor abdominal.

Lo sufren aproximadamente el $20 \%$ de las mujeres. El dolor puede producirse justo antes, durante o después de la ovulación. Hay varias explicaciones. Justo antes de la ovulación, el crecimiento del folículo puede prolongar la superficie del ovario, causando dolor. En el momento de la ovulación, líquido o sangre se libera de la ruptura del folículo y puede causar irritación del revestimiento abdominal. Puede sentirse en un lado un mes, luego hacia el lado opuesto el mes siguiente, o puede ser sen- 
tido en el mismo lado durante varios meses sucesivamente.

Es dolor abdominal inferior de características:

- Unilateral

- Recurrente o con dolor similar en los últimos meses.

- Normalmente dura de minutos a unas pocas horas, pero puede ampliar el tiempo a $24-48$ horas

- Grave (raro)

No se presenta con anomalías pélvicas aunque puede aparecer cierta irritabilidad abdominal. Pueden realizarse otros procedimientos de diagnóstico como una ecografía abdominal para descartar otras causas de dolor. Se efectúa hematimetría para valorar la posible perdida hemática.

Ningún tratamiento es necesario generalmente. Analgésicos pueden ser necesarios en casos de dolor intenso o prolongado, produciendo excelente resultado.

Normalmente no aparece ninguna complicación, aunque excepcionalmente se requiere una laparoscopia urgente para cohibir la hemorragia que produce el folículo roto.

La anticoncepción hormonal se puede tomar para prevenir la ovulación -y, por tanto el dolor ovulatorio- pero por lo demás no se conoce prevención ${ }^{27}$.

\section{DISMENORREA}

Es un dolor cíclico. Trastorno ginecológico frecuente, que afecta a cerca del $50 \%$ de las mujeres que menstrúan.

\section{Primaria}

Dolor menstrual sin trastorno pélvico que suele manifestarse 1 ó 2 años después de la menarquia -época en la que se establecen los ciclos ovulatorios-. Afecta a mujeres jóvenes pero puede persistir hasta la $5^{\text {a }}$ década. Su causa es el aumento de la producción endometrial de prostaglandinas en el endometrio secretor.

El dolor suele iniciarse unas horas antes del inicio del periodo menstrual, o justamente después del mismo, y puede durar 48-72 horas. Es de tipo cólico, suprapúbico, y se puede acompañar de dolor lumbosacro. Mejora con el masaje abdominal, el calor o los movimientos corporales.

La región suprapúbica es hipersensible a la palpación. El examen bimanual durante la crisis de dismenorrea revela hipersensibilidad uterina; no se observa dolor con la movilización cervical o al palpar anejos.

Para su diagnóstico es necesario descartar enfermedad pélvica subyacente mediante un examen ginecológico normal (dismenorrea primaria) y confirmar la naturaleza cíclica del dolor.

El tratamiento consiste en:

- Inhibidores de la síntesis de las prostaglandinas: eficaz 80\%. Deben tomarse justo antes de aparecer el dolor o al iniciarse y de forma seriada cada 6-8 horas.

- Anticonceptivos orales: disminuye la proliferación endometrial. Alivio de $>90 \%$ de casos. Tratamiento durante 4-6 meses antes de confirmar que ha fracasado.

\section{Secundaria}

Es la que aparece con trastorno pélvico subyacente y ocurre con ciclos anovulatorios; se manifiesta años después de la menarquia. Suele comenzar dos semanas antes de la menstruación y persiste unos cuantos días después de interrumpirse la hemorragia.

Las causas más frecuentes son: endometriosis, adenomiosis o portar DIU. Se trata con ACO, AINES, progestágenos y la histerectomía es el tratamiento definitivo.

\section{BIBLIOGRAFÍA}

1. Ankum, WM, Mol, BWJ, Van Der Veen, F, BosSUYT, PMM. Risk factors for ectopic pregnancy: a meta-analysis. Fertil Steril 1996; 65: 1093-1099.

2. Alsuleiman, SA, Grimes, EM. Ectopic pregnancy: a review of 147 cases. J Reprod Med 1982; 27: 101-106.

3. WeCKstein, LN. Current perspective on ectopic pregnancy. Obstet Gynecol Surv 1985; 40: 259-272.

4. Eccleston C, Kalson EA, Bell RF. Ketamine as an adjuvant to opioids for cancer pain. The cochrane database of suystematic reviews 2003 (1); CD003351. 
5. Cañete ML, García L, Cid J, Sánchez JM, Lanciego C, SÁnchez-Dehesa A. Emboliación de miomas uterinos. En: Cañete M.L. Urgencias en ginecología y obstetricia, ed. FISCAM, 2003, 659-669.

6. Kirk E, Papageorghiou AT, Condous G, Tan L, Bora $\mathrm{S}$, Bourne T. The diagnostic effectiveness of an initial transvaginal scan in detecting ectopic pregnancy. Hum Reprod 2007; 22: 2824.

7. Yao M, Tulandi T. Current status of surgical and nosurgical management of ectopic pregnancy. Fertil Steril 1997; 67: 421-433.

8. Silva C, Sammel MD, Zhou L, Gracia C, Hummel AC, BARUHART K. Human chorionic gonadotropin profile for women with ectopic pregnancy. Obstet Gynecol 2006; 107: 605-610.

9. SeEber BE, Barnhart KT. Suspected ectopic pregnancy. Obstet Gynecol 2006; 107: 399-413.

10. Kadar N, DeVore G, Romero R. Discriminatory hCG zone: its use in the sonographic evaluation for ectopic pregnancy. Obstet Gynecol 1981; 58: 156-161.

11. Poletti PA, Kinkel K, Vermeulen B, Irmav F, Unger PF, TERRIER F. Blunt abdominal trauma: should US be used to detect both free fluid and organ injuries? Radiology 2003; 227: 95-103.

12. HibBard, LT. Adnexal torsion. Am J Obstet Gynecol 1985; 152: 456-461.

13. Varras M, Tsikini A, Polyzos D, Samara Ch, HadJopoulos F, AkRIVIS $\mathrm{CH}_{\mathrm{H}}$. Uterine adnexal torsion: pathologic and gray-scale ultrasonographic findings. Clin Exp Obstet Gynecol 2004; 31: 34-38.

14. SASSo RA. Intermittent partial adnexal torsion after electrosurgical tubal ligation. J Am Assoc Gynecol Laparosc 1996; 3: 427-430.

15. Ben-Ami M, Perlitz Y, Haddad S. The effectiveness of spectral and color Doppler in predicting ovarian torsion. A prospective study. Eur J Obstet Gynecol Reprod Biol 2002; 104: 64-66.

16. ANDERs JF, Powell EC. Urgency of evaluation and outcome of acute ovarian torsion in pediatric patients. Arch Pediatr Adolesc Med 2005; 159: 532-535.
17. Oelsner G, Cohen SB, Soriano D, Admon D, MasHIACH S, CARP H. Minimal surgery for the twisted ischaemic adnexa can preserve ovarian function. Hum Reprod 2003; 18: 2599-2602.

18. Korn AP, Hessol NA, Padian NS, Bolan GA, Donegan E, LANDERS DV et al. Risk factors for plasma cell endometritis among women with cervical Neisseria gonorrhoeae, cervical Chlamydia trachomatis, or bacterial vaginosis. Am J Obstet Gynecol 1998; 178: 987-990.

19. JACOBSON L, WESTRÖM L. Objectivized diagnosis of acute pelvic inflammatory disease. Am J Obstet Gynecol 1969; 105: 1088-1098.

20. Workowski KA, Berman SM. Sexually transmitted diseases treatment guidelines, 2006. MMWR Recomm Rep 2006; 55: 1-94.

21. Eschenbach DA, Buchanan TM, Pollock HM, Forsyth PS, Alexander ER, Lin Js et al. Polymicrobial etiology of acute pelvic inflammatory disease. N Engl J Med 1975; 293: 166-171.

22. Sinail N, Plumb K, Cotton L, Lambert A, Kennedy $\mathrm{S}$, Zondervan $\mathrm{K}$ et al. Differences in characteristics among 1,000 women with endometriosis based on extent of disease. Fertil Steril 2008; 89: 538-545.

23. Porpora MG, Koninckx PR, Piazze J, Natili M, Colagrande S, Cosmi EU. Correlation between endometriosis and pelvic pain. J Am Assoc Gynecol Laparosc 1999; 6: 429-434.

24. Koninckx PR, Meuleman C, Demeyere S, Lesaffre E, Cornillie FJ. Suggestive evidence that pelvic endometriosis is a progressive disease, whereas deeply infiltrating endometriosis is associated with pelvic pain. Fertil Steril 1991; 55: 759-765.

25. Fauconnier A, Chapron C, Dubuisson JB, Vieira M, Dousset B, BRÉART G. Relation between pain symptoms and the anatomic location of deep infiltrating endometriosis. Fertil Steril 2002; 78: 719-726.

26. Kennedy S, Bergqvist A, Chapron C, D'Hooghe T, Dunselman G, GRelo R et al. ESHRE guideline for the diagnosis and treatment of endometriosis. Hum Reprod 2005; 20: 2698-2704.

27. Goldman L, Ausiello D. Cecil de libros de texto de medicina, 22 ed. Filadelfia, Pa: WB Saunders; 2004: 1495. 